OPEN ACCESS

Edited by:

Hong Ni,

Children's Hospital of Soochow

University, China

Reviewed by:

Johanna Westra,

University of Groningen, Netherlands

Giulia Freer,

University of Pisa, Italy

*Correspondence:

Jonathan D. Santoro

jdsantoro@chla.usc.edu orcid.org/0000-0002-8350-8234

Specialty section:

This article was submitted to

Pediatric Neurology

a section of the journal

Frontiers in Pediatrics

Received: 06 October 2021 Accepted: 10 November 2021 Published: 01 December 2021

Citation:

Santoro JD, Saucier LE, Tanna R,

Wiegand SE, Pagarkar D,

Tempchin AF, Khoshnood M, Ahsan N and Van Haren K (2021) Inadequate

Vaccine Responses in Children With Multiple Sclerosis.

Front. Pediatr. 9:790159.

doi: 10.3389/fped.2021.790159

\section{Inadequate Vaccine Responses in Children With Multiple Sclerosis}

\author{
Jonathan D. Santoro ${ }^{1,2 *}$, Laura E. Saucier ${ }^{3}$, Runi Tanna ${ }^{4}$, Sarah E. Wiegand ${ }^{1}$, \\ Dania Pagarkar ${ }^{2}$, Adam F. Tempchin ${ }^{2}$, Mellad Khoshnood ${ }^{1}$, Nusrat Ahsan ${ }^{1,2}$ and \\ Keith Van Haren ${ }^{5}$ \\ ${ }^{1}$ Division of Neurology, Department of Pediatrics, Children's Hospital Los Angeles, Los Angeles, CA, United States, \\ ${ }^{2}$ Department of Neurology, Keck School of Medicine, University of Southern California, Los Angeles, CA, United States, \\ ${ }^{3}$ Department of Neurology, Massachusetts General Hospital, Boston, MA, United States, ${ }^{4}$ Keck School of Medicine, \\ University of Southern California, Los Angeles, CA, United States, ${ }^{5}$ Department of Neurology, Stanford University School of \\ Medicine, Stanford, CA, United States
}

Objective: Immunizations against Hepatitis B virus (HBV) and Varicella Zoster virus (VZV), are recommended for patients with pediatric onset multiple sclerosis (POMS) and may be required prior to initiation of some disease modifying therapies. However, the efficacy of routine vaccine administration in POMS has never been studied. We sought to assess the humoral mediated vaccine response to HBV and VZV in children with POMS.

Methods: A multi-center retrospective chart-based review of 62 patients with POMS was performed. Clinical data and antibody titers against HBV and VZV were collected prior to initiation of disease modifying therapy or steroids and compared to institutional control data, using $t$-test and chi squared analysis.

Results: There were low rates of immunity against both HBV and VZV (33 and 25\% respectively) among individuals with POMS. Fifteen individuals (24\%) were non-immune to both. Compared to institutional control data, individuals with POMS were significantly less likely to be immune to and HBV $(p=0.003,95 \%$ Cl: $0.22-0.75)$ and VZV $(p<0.001$, 95\% Cl: 0.09-0.39).

Interpretation: Individuals with POMS have low rates of antibody-mediated immunity against HBV and VZV, despite receiving the appropriate vaccinations. This suggests an association between POMS and systemic immune dysregulation although further study is needed.

Keywords: multiple sclerosis, pediatric, vaccine, vaccine response, immunology and infectious diseases

\section{INTRODUCTION}

Multiple sclerosis (MS) is a chronic autoimmune and inflammatory condition that results in demyelinating lesions to the central nervous system. Onset in childhood makes up a minority of cases, ranging from 5-10\% (1). Pediatric onset multiple sclerosis (POMS) has been associated with higher rates of relapse and longitudinal accrual of motor and neurocognitive disability (2-4).

Though classically considered an autoimmune disorder, MS may be better characterized as a disease of immune dysregulation (5). Emerging evidence supports the concept of a dysregulation of immunological tolerance toward self-antigens of neuronal myelin structure in individuals with MS (6-8). Studied primarily as a driver of neuroinflammation, altered immune responses in individuals with MS may have other implications that are less frequently explored in the literature. 
While immune system integrity is typically assessed clinically, vaccine response is a well-established method of objective assessment. Several studies have evaluated a variety of vaccine responses in individuals with MS although this data has been limited to adults and those who were already on disease modifying therapy (DMT) (9-12). While the benefits of vaccine administration have been studied in adults with MS, the data on patients with POMS is less robust (13). While guidelines continue to advise routine vaccination schedules (13), it remains unclear whether younger patients who adhere to standardized vaccination schedules achieve the same level of humoral immunity as other pediatric patients without POMS. This is a highly relevant question given that protective antibody titers against varicella zoster virus (VZV) are required for safe use of certain DMTs such as fingolimod, the only FDA-approved therapy in POMS (14).

We sought to retrospectively assess humoral mediated hepatitis $\mathrm{B}$ virus (HBV) and VZV vaccine responses in a cohort of individuals with POMS in order to evaluate for evidence of immune dysregulation in individuals utilizing standard pediatric vaccine schedules. Our hypothesis was that evidence of immune dysregulation in the form of inadequate vaccine titer response would be present in individuals with POMS.

\section{MATERIALS AND METHODS}

\section{Data Availability}

De-identified data is available on request to qualified researchers pending IRB approval.

\section{Patient Selection}

This study was a retrospective, chart-based review, of patients evaluated at two academic medical centers that treat individuals with POMS. Data was reviewed from January 1, 2015, to December 31, 2020. Both sites had independent IRB approvals for the review of data. Patients were not contacted for this study nor was post-hoc lab data obtained. Demographic information, including age, race and ethnicity was collected for all subjects. Disease severity was assessed using annualized relapse rate and expanded disability severity score (EDSS). Laboratory data including CSF studies, vitamin D 25-OH, and immunologic serology were reviewed.

\section{Choice of Antibody Titer Selection}

Selection of some disease modifying therapies in POMS is dependent on appropriate vaccination response to both $\mathrm{HBV}$ and VZV (e.g., rituximab for HBV and fingolimod for VZV), thus, these titers are routinely assessed within 6 months of diagnosis.

Abbreviations: AQP4, Aquaporin-4; CDC, Centers for Disease Control; CI, Confidence Interval; CIS, Clinically Isolated Syndrome; CSF, Cerebrospinal Fluid; DMT, Disease Modifying Therapy; EDSS, Expanded Disability Severity Scale; FDA, Food and Drug Administration; HBV, Hepatitis B Virus; HIV, Human Immunodeficiency Virus; IPMSSG, International Pediatric Multiple Sclerosis Study Group; IRB, Institutional Review Board; MOG, Myelin Oligodendrocyte Glycoprotein; MS, Multiple Sclerosis; POMS, Pediatric Onset Multiple Sclerosis; RIS, Radiographically Isolate Syndrome; VZV, Varicella Zoster Virus.
EBV titers are not routinely collected as part of clinical practice and were thus unavailable.

\section{Inclusion Criteria}

Patients in this study must have been diagnosed with POMS per International Pediatric Multiple Sclerosis Study Group (IPMSSG) and McDonald's 2017 criteria $(15,16)$. Patients with either radiographically isolated syndrome (RIS) or clinically isolated syndrome (CIS) were also included in this cohort. All patients were aged 18 years or younger at the time of diagnosis and serologic testing. All patients had to have vaccine titers obtained prior to initiation of any disease modifying therapy and steroid therapy (in acute settings) to avoid interference with interpretation of results $(17,18)$.

\section{Exclusion Criteria}

Patients subsequently found to have a mimic of multiple sclerosis after initial diagnosis were excluded. Patient with aquaporin-4 (AQP4) antibodies were excluded as were those with myelin oligodendrocyte glycoprotein (MOG) antibodies titers $>1: 100$. Patients with a history of immunodeficiency, genetic disorder associated with immune dysregulation, recipients of chemotherapy or ionizing radiation, or prematurity (defined as $<36$ weeks gestation) were also excluded. Patients with long-term chronic medical disorders (e.g., sickle cell disease, chronic kidney disease, etc.), chronic infections affecting the immune system (e.g., HIV) and/or active/prior $\mathrm{HBV}$ and/or VZV infection were excluded.

\section{Definition of Immunity}

$\mathrm{HBV}$ immunity was determined by the presence of anti-HB surface antibodies and negative $\mathrm{HB}$ surface antigen and anti-HB core antibodies. Positive findings were defined by a titer level $\geq$ $10 \mathrm{mIU} / \mathrm{mL}$. VZV immunity was determined as follows: nonimmune $\leq 0.90$ antibody index (AI), equivocal $0.91-1.09 \mathrm{AI}$, and immune $\geq 1.10$ AI.

\section{Comparator Data}

To determine baseline rates of protective autoantibodies against $\mathrm{HBV}$ and VZV, we used clinical laboratory data from the labs at Children's Hospital Los Angeles and affiliated satellite clinics. All patients with either $\mathrm{HBV}$ and/or VZV immunologic tests from January 1, 2019, to December 31, 2020 were queried to assess rates of immunity in a heterogenous population. Patients in the comparator cohort were manually excluded from analysis if they had rheumatologic, immunologic, genetic, neoplastic, or gastrointestinal disorders of any type based on ICD-10 codes associated with the lab encounter. With no ability to screen patients beyond the level of demographics in the comparator data set, the sample likely contained some titers from individuals with suppressed immune systems. Repeat values from the same patients were excluded with the most recent value being utilized in all cases. Given the older median age of our cohort, vaccine 
comparator data was restricted to patients aged 12-18 years. Patients included in the comparator group were age and sex matched to enhance comparison to individuals with POMS.

\section{Confirmation of Vaccine Schedule}

Confirmation of receipt of vaccines against both HBV and VZV were performed by accessing vaccination reports through the California immunization registry (CAIR2). This was performed at the time of clinical evaluation as part of clinical standard of care before administering DMT and was not performed post-hoc. In cases where a CAIR2 query was not performed, review of vaccination records in the clinical documentation was performed. Documentation of appropriate vaccination was determined as $+/-6$ months from the United States Center for Disease Control suggested age for vaccine administration (Appendix 1) (19).

\section{Statistical Analysis}

Data was extracted to report frequencies and for continuous variables, median and interquartile ranges. For averages, standard deviations were also calculated. For comparison of differences between groups, Fishers exact $T$-test and chi squared analysis were used. Comparison of rates of immunity between our cohort and population-based sampling, a Fishers exact $T$-test was utilized. In addition, odds ratios were calculated for the likelihood of individuals with POMS having protective humoral immunity against HBV and VZV. To adjust for confounding in the setting of age and sex matching, stratified analysis using the CochranMantel-Haenszel method was performed. Statistical analysis was performed using IBM SPSS ${ }^{\circledR}$ software (v25).

\section{RESULTS}

In total, 72 patients were identified for inclusion in this study. Of those, 10 (14\%) met exclusionary criteria and 62 were ultimately included in data analysis. The most frequent reasons for exclusion were high titer MOG antibody $(6,60 \%)$ and incomplete data $(4,40 \%)$. No patients were excluded for prior HBV or VZV infections nor infectious causing immunodeficiency such as HIV. Two patients with MOG antibodies were included because their titer was 1:20 and of unclear clinical significance in both cases. No patients were excluded for deviation from standard pediatric vaccination administration. All patients $(62,100 \%)$ had three total HBV vaccinations. The majority of patients had received two total VZV vaccinations $(52,84 \%)$ with 10 patients only having one vaccination, all of whom were under 13 years of age at the time of diagnosis.

Demographic and clinical data regarding cases are presented in Table 1. Our cohort had a median age at diagnosis of 14.5 years and an average time to most recent follow up was 2.9 years. All patients had assessment of humoral immunity within 6 months of diagnosis, and all were prior to initiation of immunotherapy. Individuals were largely Caucasian (43, 70\%) although were predominantly of Hispanic/Latino descent (29, 67\%). Nearly all patients (90\%) had classic relapsing remitting type multiple sclerosis although there were two cases of tumefactive multiple sclerosis and three cases of CIS/RIS. Disability was mild, with a median EDSS score of 1.5. Patients received first line high efficacy treatments in $61 \%$ of cases and $32 \%$ of patients required escalation to a second line DMT. Only $6.4 \%$ of individuals required additional escalation of therapy.

Vaccine histories were available in all but two patients (3\%) using CAIR2. In the remaining patients, vaccines were administered at age-appropriate, CDC-guided, checkpoints in all cases +/- 6 months. Vaccination response data are presented in Table 2. A total of 46 patients had HBV immunity testing. The median HBV titer was 47.5 (IQR: 17.9-333.0) and the median time from last $\mathrm{HBV}$ vaccination was 12.4 years (IQR: 10.5$15.2)$, with all patient's receiving three total doses. Fifteen (33\%) patients were HBV immune and 31 (67\%) patients were nonimmune. A total of 39 patients had VZV immunity testing. The median VZV titer was 0.78 (IQR: 0.53-1.36) and the median time from last VZV vaccination was 2.4 years (IQR: $1.8-2.9$ ) with all patients having received two total doses. Ten (26\%) patients had VZV immunity, 5 (13\%) had equivocal immunity, and $24(63 \%)$ were non-immune. In secondary analysis, 13 of 16 (81\%) individuals of Hispanic/Latino descent were non-immune compared to 9 of $16(44 \%)$ of individuals not of Hispanic/Latino descent ( $p=0.14,95 \% \mathrm{CI}: 0.06-1.47)$. There was no effect of brand of vaccine on HBV immunity $(p=0.67,95 \% \mathrm{CI}$ : $0.58-2.87$ ) or VZV immunity ( $p=0.89,95 \% \mathrm{CI}: 0.41-5.22)$ in multivariate analysis. Vitamin $\mathrm{D}$ level at diagnosis was not predictive of immunity for either VZV or HBV $(p=0.45$ and $p=0.69$ respectively).

A total of 15 (24\%) patients were non-immune to both HBV and VZV. This group was slightly older (median age 15 years compared to 14.5 years). These non-immune individuals were more likely to be prescribed DMT that did not require HBV or VZV immunity with 12 (80\%) being placed on either dimethyl fumarate, natalizumab, or interferon. Amongst all patients with lack of vaccine response to either HBV (31), VZV (24) or both (15), only five underwent revaccination and follow up titers were not obtained in any case.

Disease severity did not impact humoral responses in this study. Individuals with high annualized relapse rate $\geq 2(n=8)$ were not more likely to have poor humoral responses to vaccines against $\mathrm{HBV}(p=0.99,95 \% \mathrm{CI}: 0.21-4.81)$ or $\mathrm{VZV}(p=0.65$, 95\% CI: 0.11-3.76). Similarly, individuals with EDSS $\geq 2(n=11)$ demonstrated had no differences in humoral immune response against HBV ( $p=0.42,95 \%$ CI: $0.41-8.55)$ or VZV ( $p=0.11$, 95\% CI: 0.01-1.76).

Between 2019 and 2020, a total of 3,285 HBV and 1,082 VZV immunity tests were performed, of which 2,309 (70\%) and 943 (87\%) met no exclusion criteria, respectively. To account for age and sex matching, these cohorts were further reduced to 2,027 individuals with $\mathrm{HBV}$ testing and 730 individuals with VZV testing. Demographics of the comparator group are provided in Table 1. Comparison between individuals without POMS and individuals with POMS are displayed in Table 2 and Figure 1. Overall rates of immunity were 55 and $65 \%$ respectively. After controlling for sex and age, rates of immunity were significantly higher in individuals without POMS compared to individuals with POMS (OR: $0.40, p=0.003,95 \%$ CI: $0.22-0.75$ for HBV and OR: $0.19, p<0.001,95 \%$ CI: $0.09-0.39$ for VZV). At the time of 
TABLE 1 | Demographic and clinic data.

\begin{tabular}{|c|c|c|c|}
\hline & $\begin{array}{l}\text { POMS } \\
n=62\end{array}$ & $\begin{array}{c}\text { Control } \\
n=2757\end{array}$ & $\begin{array}{c}p \text { Value } \\
(95 \% \mathrm{Cl})\end{array}$ \\
\hline Age at diagnosis (median, IQR) & $14.5(11-16)$ & $14(9-17)$ & $0.91(0.04-0.86)$ \\
\hline Age at present (median, IQR) & $17(15-19)$ & - & - \\
\hline Years of POMS (average, STD) & $2.91(2.61)$ & - & - \\
\hline \multicolumn{4}{|l|}{ Gender } \\
\hline Male & $23(37 \%)$ & $1048(38 \%)$ & $0.87(-1.3-1.2)$ \\
\hline Female & $39(63 \%)$ & 1709 (62\%) & \\
\hline \multicolumn{4}{|l|}{ Race } \\
\hline Caucasian & 43 (70\%) & 2012 (73\%) & \\
\hline Other/Mixed & $8(13 \%)$ & $193(7 \%)$ & \\
\hline Asian & $6(10 \%)$ & $121(4 \%)$ & $0.61(-0.72-15.4)$ \\
\hline African American & $1(1.5 \%)$ & $27(1 \%)$ & \\
\hline Native American & $1(1.5 \%)$ & $22(1 \%)$ & \\
\hline Unknown/Not disclosed & $2(3 \%)$ & $19(1 \%)$ & \\
\hline \multicolumn{4}{|l|}{ Ethnicity } \\
\hline Hispanic/Latino & $29 / 48(60 \%)$ & $\begin{array}{c}1164 / 1847 \\
(63 \%)\end{array}$ & \\
\hline Not-Hispanic/Latino & $19 / 48(40 \%)$ & $\begin{array}{c}683 / 1847 \\
(37 \%)\end{array}$ & $0.67(-0.98-17.3)$ \\
\hline Unknown/Not disclosed & $14 / 62(22 \%)$ & $\begin{array}{c}910 / 2757 \\
(33 \%)\end{array}$ & \\
\hline $\begin{array}{l}\text { Personal history of recurrent } \\
\text { infection }\end{array}$ & $7(11 \%)$ & & \\
\hline $\begin{array}{l}\text { Personal history of autoimmune } \\
\text { disorder }\end{array}$ & $3(4.5 \%)$ & & \\
\hline $\begin{array}{l}\text { 1st degree relative with } \\
\text { autoimmune disorder }\end{array}$ & $9(15 \%)$ & & \\
\hline \multicolumn{4}{|l|}{ MOG antibody status } \\
\hline Positive & $2 / 47(3 \%)$ & & \\
\hline Negative & $45 / 47(96 \%)$ & & \\
\hline Not known & 15 (24\%) & & \\
\hline \multicolumn{4}{|l|}{$\begin{array}{l}\text { Immunoglobulin status (median, } \\
\text { IQR) }\end{array}$} \\
\hline $\lg G(n=60)$ & $\begin{array}{c}1156 \\
(727-1389)\end{array}$ & & \\
\hline $\operatorname{lgM}(n=46)$ & $104(69-159)$ & & \\
\hline $\lg A(n=44)$ & 188 (94-294) & & \\
\hline Immunodeficiency present & 0/60 (0\%) & & \\
\hline \multicolumn{4}{|l|}{ CSF findings $(n=52)$} \\
\hline WBC & $3(1-12)$ & & \\
\hline \% lymphocytes & $\begin{array}{c}91 \\
(82.5-94.5)\end{array}$ & & \\
\hline Total protein & $30(23-44)$ & & \\
\hline Oligoclonal bands & $\begin{array}{l}5(2-5), 98 \% \\
\text { positive }\end{array}$ & & \\
\hline $\lg G$ index & $\begin{array}{c}0.75 \\
(0.59-1.10)\end{array}$ & & \\
\hline Neopterin $(n=15)$ & $20(14-24)$ & & \\
\hline \multicolumn{4}{|l|}{ Diagnosis } \\
\hline $\begin{array}{l}\text { Relapsing remitting multiple } \\
\text { sclerosis }\end{array}$ & $56(90 \%)$ & & \\
\hline CIS/RIS & $3(5 \%)$ & & \\
\hline Tumefactive multiple sclerosis & $2(3 \%)$ & & \\
\hline
\end{tabular}

(Continued)
TABLE 1 | Continued

\begin{tabular}{|c|c|c|c|}
\hline & $\begin{array}{l}\text { РОMS } \\
n=62\end{array}$ & $\begin{array}{c}\text { Control } \\
n=2757\end{array}$ & $\begin{array}{l}p \text { Value } \\
(95 \% \mathrm{Cl})\end{array}$ \\
\hline Atypical multiple sclerosis & $1(1.5 \%)$ & & \\
\hline \multicolumn{4}{|l|}{ Vitamin D 25-OH status } \\
\hline At diagnosis (median, IQR) & $18.0(14-22)$ & & \\
\hline After diagnosis (average, SD) & $38.8(14.16)$ & & \\
\hline $\begin{array}{l}\text { Annualized relapse rate } \\
\text { (median, IQR) }\end{array}$ & $\begin{array}{c}0.6 \\
(0.23-1.08)\end{array}$ & & \\
\hline EDSS (median, IQR) & $1.5(1.0-2.5)$ & & \\
\hline \multicolumn{4}{|l|}{ Therapy } \\
\hline High efficacy first line therapy & 38 (61\%) & & \\
\hline Failure of one DMT & 20 (32\%) & & \\
\hline Failure of $\geq 2$ DMT & $4(6.4 \%)$ & & \\
\hline
\end{tabular}

this study, no individual with inadequate humoral immunity to either VZV or HBV has contracted either virus.

\section{DISCUSSION}

This study reports insufficient humoral immune response to both HBV and VZV vaccination in children and adolescents with POMS. All individuals in our cohort had complied with routine pediatric vaccination schedules yet yielded low rates of humoral immunity, only $33 \%$ for HBV and $26 \%$ for VZV. Compared to institutional controls, which included individuals with suppressed immune systems, these results were markedly divergent. The likelihood of having protective humoral immunity against HBV and VZV was 2.5 and 5 times greater, respectively, in individuals without POMS than with those with POMS. Despite the typical designation of MS as an autoimmune disease, our data provides evidence for immune dysregulation in POMS. This finding is noteworthy not only for its potential to inform our understanding of the pathophysiology of POMS, but also given the practical implications of humoral immune dysregulation on DMT selection and risk in the pediatric population (9).

It has been established that rates of humoral vaccine immunity declines over time. Waning HBV immunity over time is well established $(20,21)$ and VZV response has been similarly shown to decline by up to $8 \%$ per year in healthy, neurotypical, individuals $(22,23)$. Although these studies highlight the lower than anticipated immunity rates in vaccinated individuals, the overall immunity rates in these studies remain much higher than what was found in our cohort. Although specific age and gender matching was not feasible in this study, control data was limited to similar age groups (12-18 years) allowing for a reasonable, although imperfect, detailing of discrepant humoral driven immunity in young persons with POMS.

Interestingly, secondary analysis revealed that individuals with POMS of Hispanic/Latino descent have even lower rates of VZV immunity (19\%) than non-Hispanic/Latino POMS patients (56\%). This finding was statistically significant although a similar phenomenon was not observed for HBV. Nevertheless, 
TABLE 2 | Vaccination data.

\begin{tabular}{|c|c|c|c|c|c|}
\hline & $\begin{array}{l}\text { POMS } \\
(n, \%)\end{array}$ & $\begin{array}{l}\text { Institutional controls } \\
\qquad(n, \%)\end{array}$ & OR & $p$ values & $95 \% \mathrm{Cl}$ \\
\hline VZV titer (median, IQR) & $0.78(0.53-1.36)$ & $1.83(0.89-2.21)$ & & & \\
\hline VZV immunity & $n=39$ & $n=730$ & 0.19 & $p<0.001$ & $0.09-0.39$ \\
\hline Immune & $10(26)$ & $473(65)$ & & & \\
\hline Equivocal & $5(13)$ & $44(6)$ & & & \\
\hline HBV immunity & $n=46$ & $n=2,027$ & 0.40 & $p=0.004$ & $0.22-0.75$ \\
\hline Immune & $15(33)$ & $1,105(55)$ & & & \\
\hline Equivocal & $0(0)$ & $18(1)$ & & & \\
\hline Non-immune & $31(67)$ & $904(44)$ & & & \\
\hline Unknown/Not tested & 16/62 (26) & $\mathrm{n} / \mathrm{a}$ & & & \\
\hline
\end{tabular}

Cl, Confidence interval; HBV, hepatitis B virus; OR, odds ratio; VZV, varicella zoster virus.

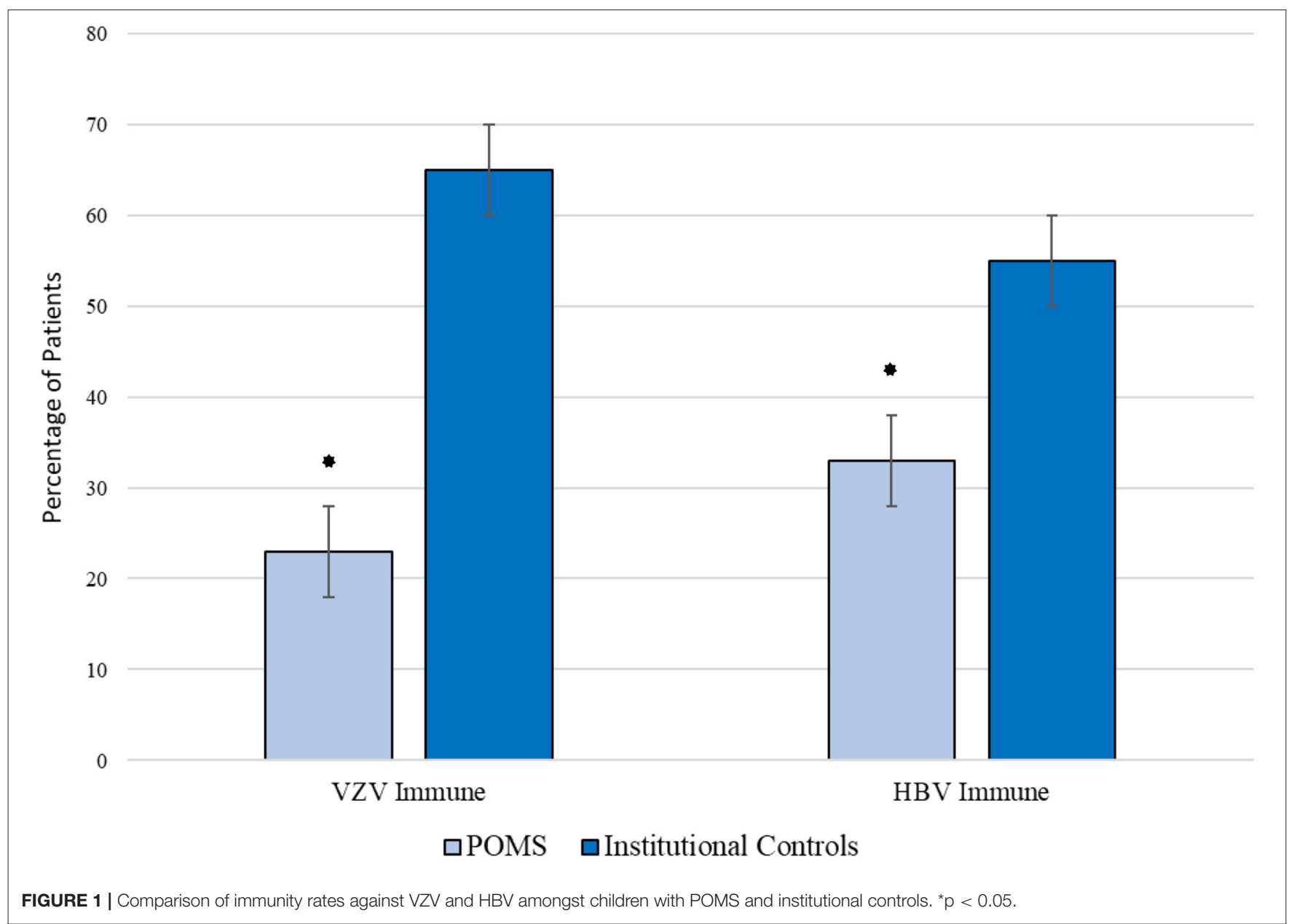

lower rates of humoral vaccine response among Hispanic/Latino individuals are notable given that more aggressive MS clinical courses have been reported in this population (24-26). Thus, one may hypothesize that more aggressive demyelinating disease might be associated with a greater degree of immune dysregulation, though multiple genetic, environmental, and treatment-related factors likely confound this connection. Further, the varied races and ethnicities in our cohort may explain discordant rates of humoral vaccine response when compared to a racially and ethnically homogenous Canadian cohorts of 
young persons with POMS (27). Although the overall humoral vaccine response was $86.7 \%$ in that study, the median age of individuals tested was nearly 2 years younger than in our cohort, possibly making direct comparison difficult, especially in the context of established waning of VZV immune response over time $(20,21)$.

This study only evaluated the humoral mediated vaccine response as measured by antibody titers. This is the type of immunity testing that is routinely utilized in clinical practice and was therefore most readily available for our retrospective analysis. Measurements of CD4+ memory T cell and CD8+ effector $\mathrm{T}$ cell responses were not included in our analysis as the data was unavailable. Cellular immunity is an important component to the immune response in both and HBV (28) and VZV (29-31) vaccination. Some studies have even suggested that "protective" antibodies may not be required in the setting of longstanding cellular immunity; however, these studies have typically been conducted in otherwise healthy individuals without other evidence of immune dysregulation (32). In the absence of protective antibody titers, isolated cellular immunity may not provide robust sterilizing immunity that may be needed in patients who are immune suppressed or are on DMT $(32,33)$. This is of particular importance in that individuals with POMS have previously been reported to have advanced chronologic age of T-cells, (34) dysfunctional T-cell reactivity, (35) and/or exaggerated pro-inflammatory Tcell milieu (36). These factors, although not assessed in this study, do raise the suspicion for the possibility of dual B and $\mathrm{T}$ cell dysfunction in vaccine response amongst individuals with POMS.

Genetic and epigenetic factors, some of which may be shared between individuals with MS and other autoimmune disorders, may attenuate the T-cell driven vaccine response as well $(37,38)$. In addition, the interface between gene regulation, cellular immunity and cytokine response, is under-explored and may impact immunity against vaccine-preventable infection (39). A major question raised by this study is if presence of immune dysregulation such as poor humoral vaccine response is present prior to the diagnosis of POMS or after the onset of symptoms. The vaccine response cascade in both HBV and VZV is complex and multi-faceted and for this reason, it would be prudent to use the data reported in this study as a springboard for additional investigation into the immune responses of individuals with POMS.

The data presented in this report is best approached in a tempered fashion as this study is not without limitations. Firstly, this is a chart-based, retrospective review. This study, while multi-center, may have limited generalizability given its regional nature and focus on tertiary academic medical centers which may result in a severity bias. One quarter of patients in this study were not tested for HBV immunity and one third were not tested for VZV immunity which reduces the total sample size for comparison and also introduces bias for which patients were tested (e.g., concern for an immune deficiency or dysregulation). Though meaningful for discussion, this is a hypothetical concern as it was not noted in clinical documentation for any in our cohort. Next, as mentioned previously, this study only assessed humoral immunity, a small component of the complex vaccine response. For some of the patients who were classified as "nonimmune" by antibody titer, we cannot exclude the possibility that they may harbor a T-cell mediated vaccine response sufficient enough to provide protection against infection. This limitation in our study highlights the importance of future investigations into T-cell mediated vaccine responses and other measures of immunity in patients with POMS, especially in the context of DMTs that may confer increased risk of vaccine-preventable illness. Finally, this study did not assess the need for or effect of re-vaccination; further study into the mechanisms of vaccine response in POMS, particularly with regard to the role of cellular immunity, should precede the evaluation of any potential intervention.

\section{CONCLUSIONS}

This study suggests that in a multi-center cohort of individuals with POMS, humoral vaccine-driven immunity against HBV and VZV is significantly lower than institutional controls despite adherence to CDC guidelines regarding vaccination schedules. Although only traditional humoral mediated titer responses were assessed in this study, the potential implications for diminished immunogenicity of protective vaccines in this vulnerable population is of extraordinary importance, especially in the context of DMT use. Further investigation in this area is both needed and warranted.

\section{DATA AVAILABILITY STATEMENT}

The datasets presented in this study can be found in online repositories. The names of the repository/repositories and accession number(s) can be found in the article/Supplementary Material.

\section{ETHICS STATEMENT}

The studies involving human participants were reviewed and approved by Children's Hospital Los Angeles. Written informed consent to participate in this study was provided by the participants' legal guardian/next of kin.

\section{AUTHOR CONTRIBUTIONS}

JS was responsible for drafting, revision of the manuscript for content, including medical writing, study concept, design, analysis and interpretation of data, and had a major role in the acquisition of data. LS was responsible for drafting, revision of the manuscript for content and had a major role in the acquisition of data. RT, DP, and AT was responsible for acquisition of the data and analysis and interpretation of the data. SW performed primary and secondary analysis and interpretation of the data and all statistical analysis. MK played a major role in the acquisition of the data and assisted with the interpretation of data. NA was responsible for study concept and design as well as editing for intellectual content. 
KV was responsible for drafting, revision of the manuscript for content, including medical writing for content, study concept, design, and analysis and interpretation of data. All authors contributed to the article and approved the submitted version.

\section{REFERENCES}

1. Renoux C, Vukusic S, Mikaeloff Y, Edan G, Clanet M, Dubois B, et al. Natural history of multiple sclerosis with childhood onset. N Engl J Med. (2007) 356:2603-13. doi: 10.1056/NEJMoa067597

2. Gorman MP, Healy BC, Polgar-Turcsanyi M, Chitnis T. Increased relapse rate in pediatric-onset compared with adult-onset multiple sclerosis. Arch Neurol. (2009) 66:54-9. doi: 10.1001/archneurol.2008.505

3. Benson LA, Healy BC, Gorman MP, Baruch NF, Gholipour T, Musallam A, et al. Elevated relapse rates in pediatric compared to adult MS persist for at least 6 years. Mult Scler Relat Disord. (2014) 3:18693. doi: 10.1016/j.msard.2013.06.004

4. Santoro JD, Waltz M, Aaen G, Belman A, Benson L, Gorman M, et al. Pediatric multiple sclerosis severity score in a large US cohort. Neurology. (2020) 95:e1844-53. doi: 10.1212/WNL.0000000000010414

5. Cavallo S. Immune-mediated genesis of multiple sclerosis. J Transl Autoimmun. (2020) 3:100039. doi: 10.1016/j.jtauto.2020.100039

6. Fletcher JM, Lalor SJ, Sweeney CM, Tubridy N, Mills KHG. T cells in multiple sclerosis and experimental autoimmune encephalomyelitis. Clin Exp Immunol. (2010) 162:1-11. doi: 10.1111/j.1365-2249.2010.04143.x

7. Magliozzi R, Howell O, Vora A, Serafini B, Nicholas R, Puopolo M, et al. Meningeal B-cell follicles in secondary progressive multiple sclerosis associate with early onset of disease and severe cortical pathology. Brain. (2007) 130:1089-104. doi: 10.1093/brain/awm038

8. Genain CP, Cannella B, Hauser SL, Raine CS. Identification of autoantibodies associated with myelin damage in multiple sclerosis. Nat Med. (1999) 5:1705. doi: $10.1038 / 5532$

9. Winkelmann A, Loebermann M, Reisinger EC, Hartung HP, Zettl UK. Disease-modifying therapies and infectious risks in multiple sclerosis. Nat Rev Neurol. (2016) 12:217-33. doi: 10.1038/nrneurol.2016.21

10. Olberg HK, Cox RJ, Nostbakken JK, Aarseth JH, Vedeler CA, Myhr KM. Immunotherapies influence the influenza vaccination response in multiple sclerosis patients: an explorative study. Mult Scler. (2014) 20:107480. doi: 10.1177/1352458513513970

11. Olberg HK Eide GE, Cox RJ, Jul-Larsen A, Lartey SL, Vedeler CA, et al. Antibody response to seasonal influenza vaccination in patients with multiple sclerosis receiving immunomodulatory therapy. Eur J Neurol. (2018) 25:52734. doi: 10.1111/ene.13537

12. Metze C, Winkelmann A, Loebermann M, Hecker M, Schweiger B, Reisinger EC, et al. Immunogenicity and predictors of response to a single dose trivalent seasonal influenza vaccine in multiple sclerosis patients receiving disease-modifying therapies. CNS Neurosci Ther. (2019) 25:24554. doi: $10.1111 / \mathrm{cns} .13034$

13. Farez MF, Correale J, Armstrong MJ, Rae-Grant A, Gloss D, Donley $\mathrm{D}$, et al. Practice guideline update summary: Vaccine-preventable infections and immunization in multiple sclerosis: Report of the Guideline Development, Dissemination, and Implementation Subcommittee of the American Academy of Neurology. Neurology. (2019) 93:584-94. doi: 10.1212/WNL.0000000000008157

14. Chitnis T, Arnold DL, Banwell B, Bruck W, Ghezzi A, Giovannoni G, et al. Trial of fingolimod versus interferon beta-1a in pediatric multiple sclerosis. $N$ Engl J Med. (2018) 379:1017-27. doi: 10.1056/NEJMoa1800149

15. Thompson AJ, Banwell BL, Barkhof F, Carroll WM, Coetzee T, Comi G, et al. Diagnosis of multiple sclerosis: 2017 revisions of the McDonald criteria. Lancet Neurol. (2018) 17:162-73. doi: 10.1016/S1474-4422(17) 30470-2

16. Krupp LB, Tardieu M, Amato MP, Banwell B, Chitnis T, Dale RC, et al. International Pediatric Multiple Sclerosis Study Group criteria for pediatric multiple sclerosis and immune-mediated central nervous system

\section{SUPPLEMENTARY MATERIAL}

The Supplementary Material for this article can be found online at: https://www.frontiersin.org/articles/10.3389/fped. 2021.790159/full\#supplementary-material

demyelinating disorders: revisions to the 2007 definitions. Mult Scler. (2013) 19:1261-7. doi: 10.1177/1352458513484547

17. Settipane GA, Pudupakkam RK, McGowan JH. Corticosteroid effect on immunoglobulins. J Allergy Clin Immunol. (1978) 62:162-6. doi: 10.1016/0091-6749(78)90101-X

18. Ciotti JR, Valtcheva MV, Cross AH. Effects of MS disease-modifying therapies on responses to vaccinations: A review. Mult Scler Relat Disord. (2020) 45:102439. doi: 10.1016/j.msard.2020.102439

19. Wodi AP, Ault K, Hunter P, McNally V, Szilagyi PG, Bernstein H. Advisory committee on immunization practices recommended immunization schedule for children and adolescents aged 18 years or younger United States, 2021. MMWR Morb Mortal Wkly Rep. (2021) 70:18992. doi: 10.15585/mmwr.mm7006al

20. Stefanati A, Bolognesi N, Sandri F, Dini G, Massa E, Montecucco A, et al. Long-term persistency of hepatitis B immunity: an observational crosssectional study on medical students and resident doctors. J Prev Med Hyg. (2019) 60:E184-e190. doi: 10.15167/2421-4248/jpmh2019.60.3.1315

21. Dini G, Toletone A, Barberis I, Debarbieri N, Massa E, Paganino C, et al. Persistence of protective anti-HBs antibody levels and anamnestic response to HBV booster vaccination: A cross-sectional study among healthcare students 20 years following the universal immunization campaign in Italy. Hum Vaccin Immunother. (2017) 13:440-4. doi: 10.1080/21645515.2017.1264788

22. Duncan JR, Witkop CT, Webber BJ, Costello AA. Varicella seroepidemiology in United States air force recruits: A retrospective cohort study comparing immunogenicity of varicella vaccination and natural infection. Vaccine. (2017) 35:2351-7. doi: 10.1016/j.vaccine.2017.03.054

23. Grotto I, Balicer RD, Smetana Z, Davidovitch N, Bar-Zeev Y, Mendelson $\mathrm{E}$, et al. Immunity to varicella zoster virus among young adults: a decline prior to widespread uptake of varicella vaccines. Infection. (2008) 36:1304. doi: 10.1007/s15010-007-7192-7

24. Amezcua L, Lund BT, Weiner LP, Islam T. Multiple sclerosis in Hispanics: a study of clinical disease expression. Mult Scler. (2011) 17:10106. doi: 10.1177/1352458511403025

25. Amezcua L, Conti DV, Liu L, Ledezma K, Langer-Gould AM. Place of birth,age of immigration, and disability in Hispanics with multiple sclerosis. Mult Scler Relat Disord. (2015) 4:25-30. doi: 10.1016/j.msard.2014. 11.008

26. Langille MM, Islam T, Burnett M, Amezcua L. Clinical characteristics of pediatric-onset and adult-onset multiple sclerosis in hispanic Americans. $J$ Child Neurol. (2016) 31:1068-73. doi: 10.1177/0883073816638754

27. Makhani N, Banwell B, Tellier R, Yea C, McGovern S, O’Mahony J, et al. Viral exposures and MS outcome in a prospective cohort of children with acquired demyelination. Mult Scler. (2016) 22:385-8. doi: 10.1177/1352458515595876

28. Krawczyk A, Ludwig C, Jochum C, Fiedler M, Heinemann FM, Shouval $\mathrm{D}$, et al. Induction of a robust $\mathrm{T}$ - and $\mathrm{B}$-cell immune response in non- and low-responders to conventional vaccination against hepatitis B by using a third generation PreS/S vaccine. Vaccine. (2014) 32:507782. doi: 10.1016/j.vaccine.2014.06.076

29. Weinberg A, Canniff J, Rouphael N, Mehta A, Mulligan M, Whitaker JA, et al. Varicella-zoster virus-specific cellular immune responses to the live attenuated zoster vaccine in young and older adults. J Immunol. (2017) 199:604-12. doi: 10.4049/jimmunol.1700290

30. Eberhardt CS, Wieland A, Nasti TH, Grifoni A, Wilson E, Schmid DS, et al. Persistence of varicella-zoster virus-specific plasma cells in adult human bone marrow following childhood vaccination. J Virol. (2020) 94:e0212719. doi: 10.1128/JVI.02127-19

31. Laing KJ, Russell RM, Dong L, Schmid DS, Stern M, Magaret A, et al. Zoster vaccination increases the breadth of $\mathrm{CD} 4+\mathrm{T}$ cells responsive to varicella zoster virus. J Infect Dis. (2015) 212:1022-31. doi: 10.1093/infdis/jiv164 
32. Simons BC, Spradling PR, Bruden DJT, Zanis C, Case S, Chormanski TL, et al. A longitudinal hepatitis $\mathrm{B}$ vaccine cohort demonstrates long-lasting hepatitis $B$ virus (HBV) cellular immunity despite loss of antibody against HBV surface antigen. J Infect Dis. (2016) 214:273-80. doi: 10.1093/infdis/jiw142

33. Werner JM, Abdalla A, Gara N, Ghany MG, Rehermann B. The hepatitis B vaccine protects re-exposed health care workers, but does not provide sterilizing immunity. Gastroenterology. (2013) 145:102634. doi: 10.1053/j.gastro.2013.07.044

34. Balint B, Haas J, Schwarz A, Jarius S, Furwentsches A, Engelhardt K, et al. T-cell homeostasis in pediatric multiple sclerosis: old cells in young patients. Neurology. (2013) 81:784-92. doi: 10.1212/WNL.0b013e3182a2ce0e

35. Banwell B, Bar-Or A, Cheung R, Kennedy J, Krupp LB, Becker DJ, et al. Abnormal T-cell reactivities in childhood inflammatory demyelinating disease and type 1 diabetes. Ann Neurol. (2008) 63:98-111. doi: 10.1002/ana.21244

36. Mexhitaj I, Nyirenda MH Li R, O’Mahoney J, Rezk A, Rozenberg A, et al. Abnormal effector and regulatory $\mathrm{T}$ cell subsets in paediatric-onset multiple sclerosis. Brain. (2019) 142:617-32. doi: 10.1093/brain/awz017

37. Meysman P, De Neuter N, Bartholomeus E, Elias G, Van den Bergh J, Emonds MP, et al. Increased herpes zoster risk associated with poor HLA-A immediate early 62 protein (IE62) affinity. Immunogenetics. (2018) 70:36372. doi: 10.1007/s00251-017-1047-x

38. Chan VS. Epigenetics in multiple sclerosis. Adv Exp Med Biol. (2020) 1253:309-74. doi: 10.1007/978-981-15-3449-2_12
39. Hu D, Notarbartolo S, Croonenborghs T, Patel B, Cialic R, Yang TH, et al. Transcriptional signature of human pro-inflammatory $\mathrm{T}(\mathrm{H}) 17$ cells identifies reduced IL10 gene expression in multiple sclerosis. Nat Commun. (2017) 8:1600. doi: 10.1038/s41467-017-01571-8

Conflict of Interest: The authors declare that the research was conducted in the absence of any commercial or financial relationships that could be construed as a potential conflict of interest.

Publisher's Note: All claims expressed in this article are solely those of the authors and do not necessarily represent those of their affiliated organizations, or those of the publisher, the editors and the reviewers. Any product that may be evaluated in this article, or claim that may be made by its manufacturer, is not guaranteed or endorsed by the publisher.

Copyright (c) 2021 Santoro, Saucier, Tanna, Wiegand, Pagarkar, Tempchin, Khoshnood, Ahsan and Van Haren. This is an open-access article distributed under the terms of the Creative Commons Attribution License (CC BY). The use, distribution or reproduction in other forums is permitted, provided the original author(s) and the copyright owner(s) are credited and that the original publication in this journal is cited, in accordance with accepted academic practice. No use, distribution or reproduction is permitted which does not comply with these terms. 